\title{
Chinese Tabidia, 1880 (Lepidoptera: Crambidae, Spilomelinae), with description of one new species
}

\author{
Xicui Du \& Houhun Li*
}

Du, X. \& Houhun, L. 2014: Chinese Tabidia, 1880 (Lepidoptera: Crambidae, Spilomelinae), with description of one new species. — Entomol. Fennica 25: 57 64.

Three species of the genus Tabidia Snellen are recorded from China, along with a key to their identification. Among them, T. obvia sp. n. is described as new and T. strigiferalis Hampson and T. candidalis (Warren) are redescribed, including descriptions of both male and female genitalia of $T$. candidalis for the first time. The differences between T. obvia sp. n. and T. strigiferalis are diagnosed. Images of adults and genitalia are provided.

X. Du, Lepidoptera Lab, College of Life Sciences, Nankai University, Tianjin 300071, P.R. China, and College of Plant Protection, Southwest University, Chongqing 400716,P.R. China; E-mail:duxicui@hotmail.com

H. Li, Lepidoptera Lab, College of Life Sciences, Nankai University, Tianjin 300071, P.R. China; *Corresponding author's e-mail:lihouhun@nankai.edu.cn

Received 8 August 2013, accepted 11 December 2013

\section{Introduction}

Tabidia was defined by Snellen with Tabidia insanalis Snellen, 1880 as the type species. It consists of nine species mostly occurring in the Oriental and Australian regions. Tabidia strigiferalis Hampson, 1900 has been recorded from both the Palaearctic and Oriental regions. Tabidia strigiferalis and T. candidalis (Warren, 1896) have been recorded from China (Snellen 1883, Warren 1896, Hampson 1896, 1898, Caradja 1925, Caradja \& Meyrick 1933, Klima 1939, Lu \& Guan 1953, Park 1983, Kirpichnikova 1999, Song 2003, Bae et al. 2008).

The original descriptions of the two known species from China are incomplete. For example, in the original descriptions the antenna, labial palp, maxillary palp, patagium and tegula were not described, and the abdomen was barely described. After examining many specimens, including the type of $T$. candidalis, we redescribe the two known species and describe one new species from China. Moreover, male and female genitalia of $T$. candidalis are described and illustrated for the first time and those of $T$. strigiferalis are described in detail in English for the first time.

\section{Material and methods}

Specimens of adults from China were collected by light trap. Methods of genitalia dissection follow Li and Zheng (1996). Digital images of adults and genitalia were captured with Olympus and Canon digital cameras. Terminology mainly follows Maes (1995) and Kristensen (2003).

The specimens examined are deposited in the Insect Collection, College of Life Sciences, Nankai University, Tianjin (NKUM), College of Plant Protection, Southwest University, Chongqing (SWUCPP), China, and the Natural History Museum, London, UK (BMNH). The holotype 

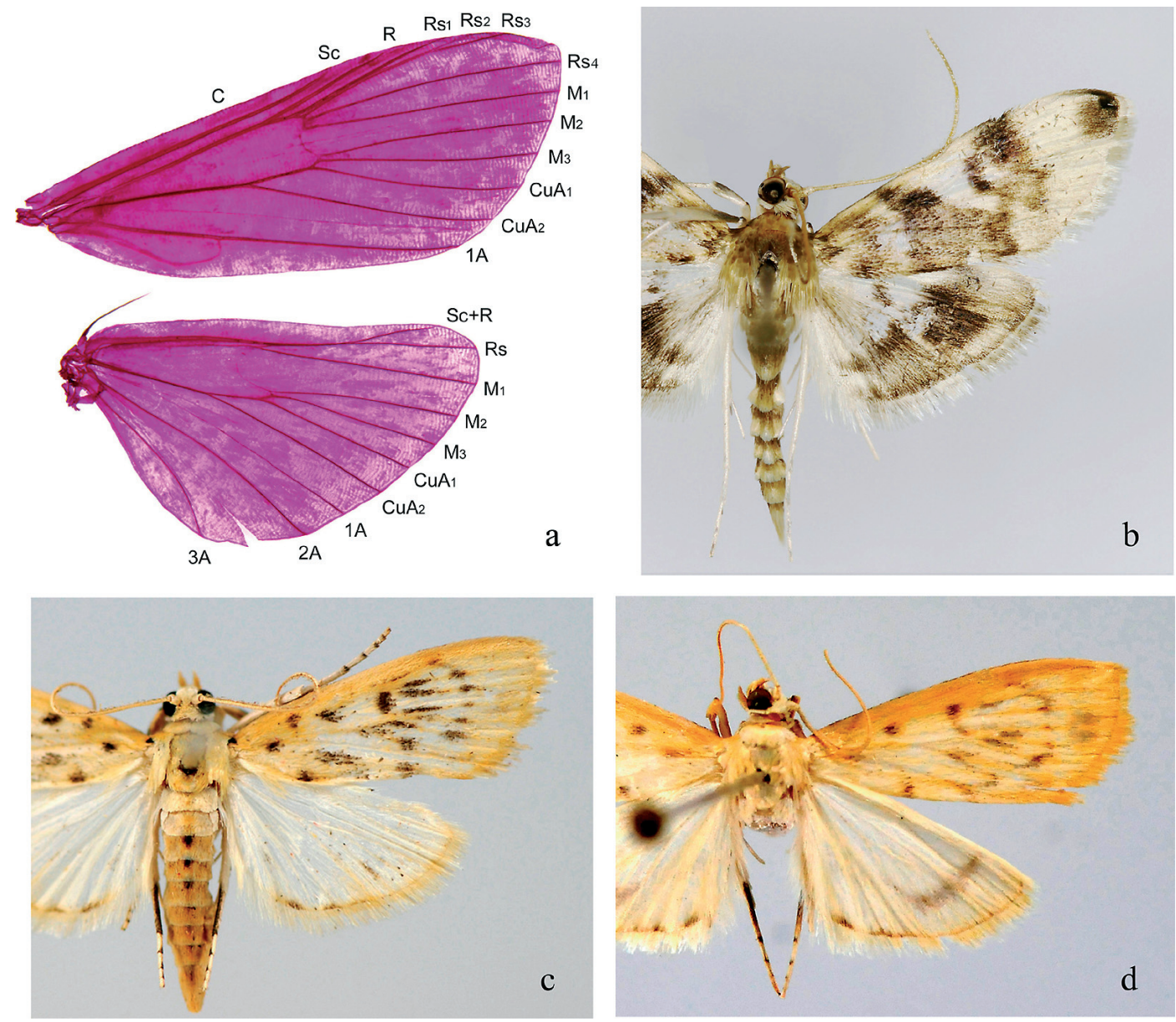

Fig. 1. Wing venation and adults of Tabidia spp. - a. Wing venation of $T$. strigiferalis Hampson. $-\mathrm{b}$. T. candidalis (Warren) male. - c. T. strigiferalis Hampson male. - d. T. obvia sp. n. male.

and eight paratypes of the new species are deposited in NKUM, and other paratypes are deposited in SWUCPP, China.

\section{Taxonomy}

\subsection{Tabidia Snellen, 1880}

Tabidia Snellen, 1880, Tijds. Ent. 23: 219.

Type species: Tabidia insanalis Snellen 1880

Generic characters. Frons rounded. Labial palp upturned and curved, $3^{\text {rd }}$ segment long, acuminate. Maxillary palp minute, filiform. Male antenna ciliate ventrally. Forewing with $\mathrm{CuA}_{1}$, $M_{3}$ and $M_{2}$ separately from posterior angle of discal cell, $\mathrm{Rs}_{4}$ straight and well separated from $\mathrm{Rs}_{2+3}, \mathrm{Rs}_{1}$ approximate to $\mathrm{Rs}_{2+3}$. Hindwing with $\mathrm{CuA}_{1}, \mathrm{M}_{3}$ and $\mathrm{M}_{2}$ from posterior angle of discal cell, $\mathrm{M}_{1}$ and Rs from anterior angle of discal cell (Fig. 1a).

Distribution. China, Korea, Russia (Far East), India, Sri Lanka, Malaysia, Indonesia, Australia, New Guinea.

Remarks. In Hampson's (1896) generic description he states that the male forewing has a dorsally located group of recumbent large scales from the base of the median nervure. We found it occurs in T. insanalis Snellen, T. aculealis Walker and $T$. craterodes Meyrick, but in T. candidalis (Warren), T. fuscifusalis Hampson, T. strigiferalis Hampson, and T. obvia sp. n. described herein, the male forewings have no groups of large 


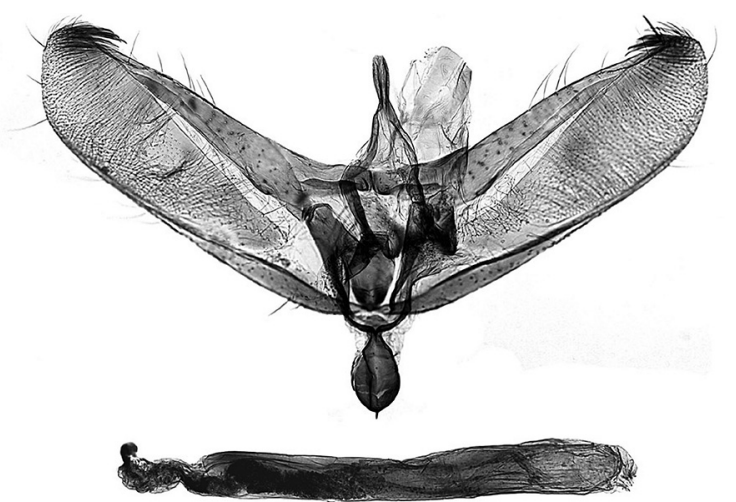

a

Fig. 2. Genitalia of Tabidia candidalis (Warren). - a. Male, slide 23157, BMNH Pyr., London. - b. Female, slide HGQ12095.

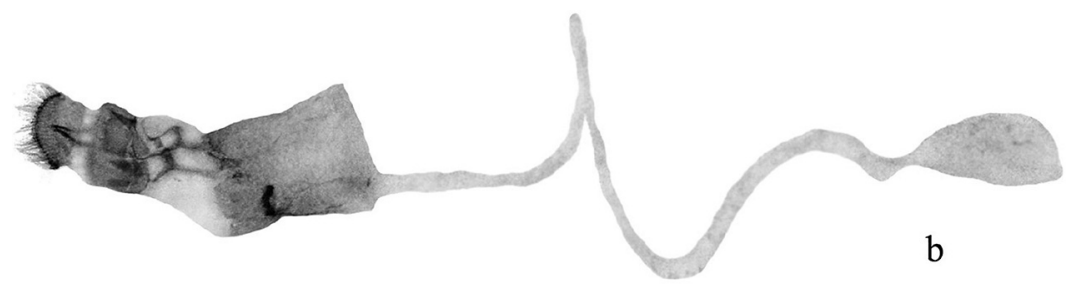

scales. The point at which $\mathrm{CuA}_{2}$ of the forewing arises differs in the genus. It is close to the base of the discal cell in the type species, near two-thirds of the discal cell in T. aculealis, and near threefourths of the distal cell in T. strigiferalis and $T$. obvia sp. n. (Fig. 1a).

\subsection{Tabidia candidalis (Warren, 1896)}

Fig. 1b, Figs. 2a-b

Aripana ? candidalis Warren, 1896, Ann. Mag. Nat. Hist. (ser. 6) 18: 169.

Tabidia candidalis Hampson, 1898, Proc. Zool. Soc. Lond. 1898: 624.

Material examined. Type. +, INDIA: Khasis, X.1895, leg. native collector. Other specimens: INDIA: Khasis, 1 §, II.1894, 1 §, XII.1895, 2 స̂े, III.1897, leg. native collector. CHINA: 2 ふ઼’’, Tianmu Mountain $\left(30^{\circ} 19^{\prime} \mathrm{N}, 119^{\circ} 26^{\prime} \mathrm{E}\right)$, Zhejiang Province, 400 m, 31.VII, 1.VIII.2011, leg. Xicui $\mathrm{Du} \&$ Xiaobing $\mathrm{Fu} ; 1$ ô, Tianmu Mountain (30¹9'N, 119²6'E), Zhejiang Province, 1,500 m, 26.VII.2011, leg. Xicui Du \& Xiaobing Fu; 29 ๙ิे, 1 q, Jiulong Mountain $\left(28^{\circ} 21^{\prime} \mathrm{N}, 118^{\circ} 52^{\prime} \mathrm{E}\right)$, Zhejiang Province, 4.7.VIII.2011, leg. Xiaobing Fu; $4 \hat{\jmath} \widehat{\jmath}$, Qing- liangfeng $\left(30^{\circ} 07^{\prime} \mathrm{N}, 118^{\circ} 54^{\prime} \mathrm{E}\right)$, Zhejiang Province, $300 \mathrm{~m}, 18 .-20$. V.2012, leg. Xiaobing Fu; 1 ふै, Nanling $\left(24^{\circ} 45^{\prime} \mathrm{N}, 112^{\circ} 51^{\prime} \mathrm{E}\right)$, Guangdong Province, 1,070 m, 23.VIII.2010, leg. Xicui Du.

Redescription. Adult (Fig. 1b): Wingspan 15.0-18.0 mm. Body white. Antenna yellowishwhite except scape tinged with fuscous laterally. Male antenna with ventral cilia slightly longer than the diameter of flagellomere. Labial palp white except $2^{\text {nd }}$ segment tinged with fuscous distally. Maxillary palp slim, white. Patagium, tegula and thorax white, except tegula tinged with black and metathorax black dorsally. Forewing white, basal area interruptedly tinged with greyish-fuscous; antemedial line blackish, slightly excurved, its outer margin with a black blotch along upper half; discoidal stigma elongate, black; postmedial line fuscous, followed on costa by a large black blotch, acutely excurved and forming a projecting beak between $\mathrm{M}_{2}$ and $\mathrm{Cu} \mathrm{A}_{1}$; lower third of median area fulvous; with a round blackish apical spot; cilia white, with a blackish basal line at apex. Hindwing white, with outer third interruptedly blackish, white ground-colour forming a small sinus in middle; discoidal stigma blackish, indistinct in some specimens; cilia white, with yellowish base and darker dividing 
line. Legs white, slightly dark on inner side of fore femur and on distal fore tibia. Abdomen white, four basal segments tinged with greyish-black dorsally; in the type specimen, basal two segments of dorsal abdomen tinged with a few greyish-black, 3-5 segments greyish-black, anal segment with basal half greyish-black.

Male genitalia (Fig. 2a). Uncus rod-like, slightly inflated laterally. Valva tongue-shaped, with dense fine hairs, terminally with thicker hairs. Costa broad, gradually narrowed towards apex. Sacculus distinctly narrower than costa. Saccus oval, contracted posteriorly, with a small projection on anterior margin. Juxta oval. Phallus cylindrical.

Female genitalia (Fig. 2b). Apophysis anterioris about 1.5 times length of apophysis posterioris, expanded and acutely projected at about basal 1/3. Ductus bursae long and thin. Corpus bursae small, oval.

Distribution. China (Zhejiang, Guangdong), India.

Biology. Life history and juvenile stages unknown.

Remarks. Warren (1896) described this species from Khasis in India based on one female specimen. It has been listed in some works (Hampson 1898, Caradja 1925, Caradja \& Meyrick 1933, Klima 1939, Lu \& Guan 1953), but has not been further morphologically studied since Warren's treatment. It was recorded from Guangdong in China by Caradja (1925) and Caradja \& Meyrick (1933), with no further information. The first author examined the type specimen in BMNH, and collected additional specimens from Zhejiang and Guangdong provinces in China.

\subsection{Tabidia strigiferalis Hampson, 1900}

Fig. 1c, Figs. 3a-b

Tabidia strigiferalis Hampson, 1900, Tr. Ent. Soc. Lond. 48: 386, pl. III, f. 30.

Material examined. CHINA: 2 9 , Faku (42 $\left.30^{\prime} \mathrm{N}, 123^{\circ} 24^{\prime} \mathrm{E}\right)$, Liaoning Province, 27.28.VII.2006, leg. Yiping Wang; 3 ふึત, 3 우, Faku (42 $\left.30^{\circ} \mathrm{N}, 123^{\circ} 24^{\prime} \mathrm{E}\right)$, Liaoning Province,

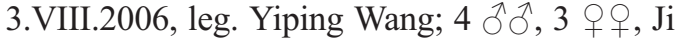
Xian $\left(40^{\circ} 02^{\prime} \mathrm{N}, 117^{\circ} 24^{\prime} \mathrm{E}\right)$, Tianjin, $550 \mathrm{~m}$,
24.VI.2001, leg. Houhun Li et al.; 1 ふૈ, 2 우, Ji Xian $\left(40^{\circ} 02^{\prime} \mathrm{N}, 117^{\circ} 24^{\prime} \mathrm{E}\right)$, Tianjin, $180 \mathrm{~m}$, 10.VI.2004, leg. Houhun Li et al.; 1 ô, Luannan County (39 $\left.30^{\prime} \mathrm{N}, 118^{\circ} 40^{\prime} \mathrm{E}\right)$, Hebei Province, 29 m, 5.VII.2001, leg. Yanli Du \& Shulian Hao; 2 ふ઼ે, Zunhua $\left(40^{\circ} 11^{\prime} \mathrm{N}, 117^{\circ} 57^{\prime} \mathrm{E}\right)$, Hebei Province, $120 \mathrm{~m}$, 6.VII.2001, leg. Yanli Du \& Shulian Hao; $1{ }^{\top}$, Zunhua $\left(40^{\circ} 11^{\prime} \mathrm{N}, 117^{\circ} 57^{\prime} \mathrm{E}\right)$, Hebei Province, 10.VII.2001, leg. Yanli Du \& Shulian Hao; $2 \partial^{\lambda}$, She Xian $\left(36^{\circ} 34^{\prime} \mathrm{N}\right.$, $\left.113^{\circ} 40^{\prime} \mathrm{E}\right)$, Hebei Province, $700 \mathrm{~m}, 1$.VIII.2000,

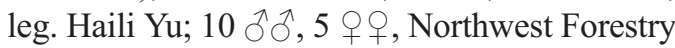
University, (341'서 $\left.108^{\circ} 04^{\prime} E\right)$, Shaanxi, Province, 3.V.-1.VI.1985, leg. Houhun Li; 1 , Northwest Forestry University, (34ำ $16^{\prime} \mathrm{N}, 108^{\circ}$ 04'E), Shaanxi Province, 27.V.1996, leg. unknown; 2 우, Northwest Agriculture University (34ำ' N, $\left.108^{\circ} 04^{\prime} \mathrm{E}\right)$, Shaanxi Province, 2.VI. 1992, leg. unknown; 3 우, Yangling $\left(34^{\circ} 16^{\prime} \mathrm{N}\right.$, 10804'E), Shaanxi Province, 6.-31.V.1993, leg. Houhun Li; 1 ㅇ, Yangling $\left(34^{\circ} 16^{\prime} \mathrm{N}, 108^{\circ} 04^{\prime} \mathrm{E}\right)$, Shaanxi Province, 450 m, 20.VI.1994, leg. Houhun Li; 4 우, Yangling $\left(34^{\circ} 16^{\prime} \mathrm{N}, 108^{\circ}\right.$ 04'E), Shaanxi Province, 450 m, 4.-14.V.1995,

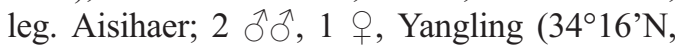
$\left.108^{\circ} 04^{\prime} \mathrm{E}\right)$, Shaanxi Province, $450 \mathrm{~m}, 16 .-21 . \mathrm{V}$. 1995, leg. Duokenli; 1 , , ningshan, Shaanxi Province, 1,360 m, 1.VII. 2007, leg. Haili Yu; 1 సَ, Wen County (32 $\left.58^{\circ} \mathrm{N}, 104^{\circ} 41^{\prime} \mathrm{E}\right)$, Gansu Pro-

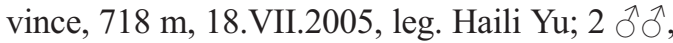
Wen County (32 $\left.58^{\prime} \mathrm{N}, 104^{\circ} 41^{\prime} \mathrm{E}\right)$, Gansu Province, 860 m, 9.-14.VII. 2005, leg. Haili Yu; 1 ō, 2 우옹 Tianshui $\left(34^{\circ} 37^{\prime} \mathrm{N}, 105^{\circ} 42^{\prime} \mathrm{E}\right)$, Gansu Province, 1,331 m, 30.VII. 2006, leg. Xinpu Wang \& Xiangfeng Shi; 3 $\widehat{\partial}$, Tongbai County $\left(32^{\circ} 21^{\prime} \mathrm{N}, 113^{\circ} 24^{\prime} \mathrm{E}\right)$, Henan Province, $300 \mathrm{~m}$, 16.VII.2001, leg. Dandan Zhang; 1 q, Dengfeng $\left(34^{\circ} 31^{\prime} \mathrm{N}, 113^{\circ} 0 ’ \mathrm{E}\right)$, Henan Provinec, $700 \mathrm{~m}$, 15.VII.2002, leg. Xinpu Wang; $1 \stackrel{\partial}{\lambda}$, Neixiang County $\left(33^{\circ} 02^{\prime} \mathrm{N}, 111^{\circ} 50^{\prime} \mathrm{E}\right)$, Henan Province, 1,200 m, 23.V.2006, leg. Xu Zhang \& Jinmei Li; $1 \partial^{\top}$, Linzhou $\left(36^{\circ} 04^{\prime} \mathrm{N}, 113^{\circ} 48^{\prime} \mathrm{E}\right)$, Henan Province, $550 \mathrm{~m}$, 21.VII.2006, leg. Hui Zhen \& Denghui Kuang; $1 \delta^{\lambda}$, Lushi County $\left(34^{\circ} 03^{\prime} \mathrm{N}\right.$, $\left.111^{\circ} 02^{\prime} \mathrm{E}\right), \quad H e n a n$ Province, $1,100 \mathrm{~m}$, 8.VIII.2006, leg. Hui Zhen \& Denghui Kuang; 21

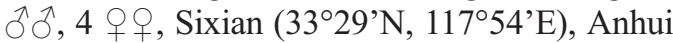
Province, 15.VIII.1999, leg. Jialiang Zhang; 6 ồ, Huangshan $\left(30^{\circ} 05^{\prime} \mathrm{N}, 118^{\circ} 10^{\prime} \mathrm{E}\right)$, Anhui Province, 6-7.VIII.2004, leg. Jiasheng Xu \& 


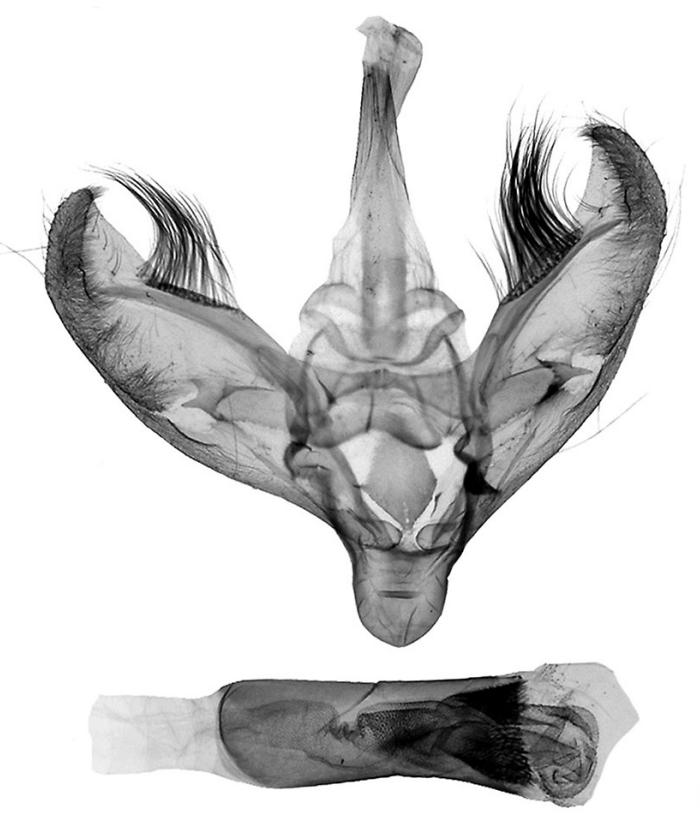

a

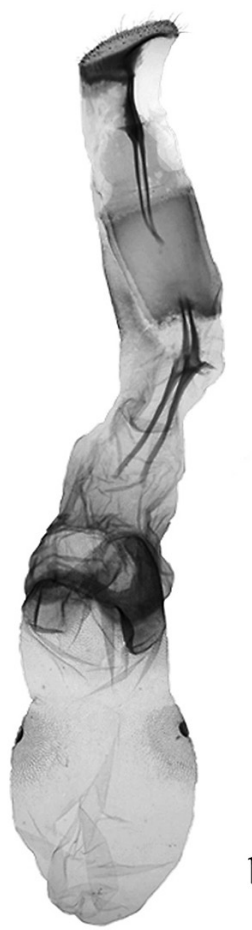

Fig. 3. Genitalia of Tabidia spp. - a. T. strigiferalis Hampson male, slide DXC05031.

- b. T. strigiferalis Hampson female, slide DXC05032. - c. T. obvia sp. n. male, slide DXC07196. - d. T. obvia sp. n. female, slide DXC07249.
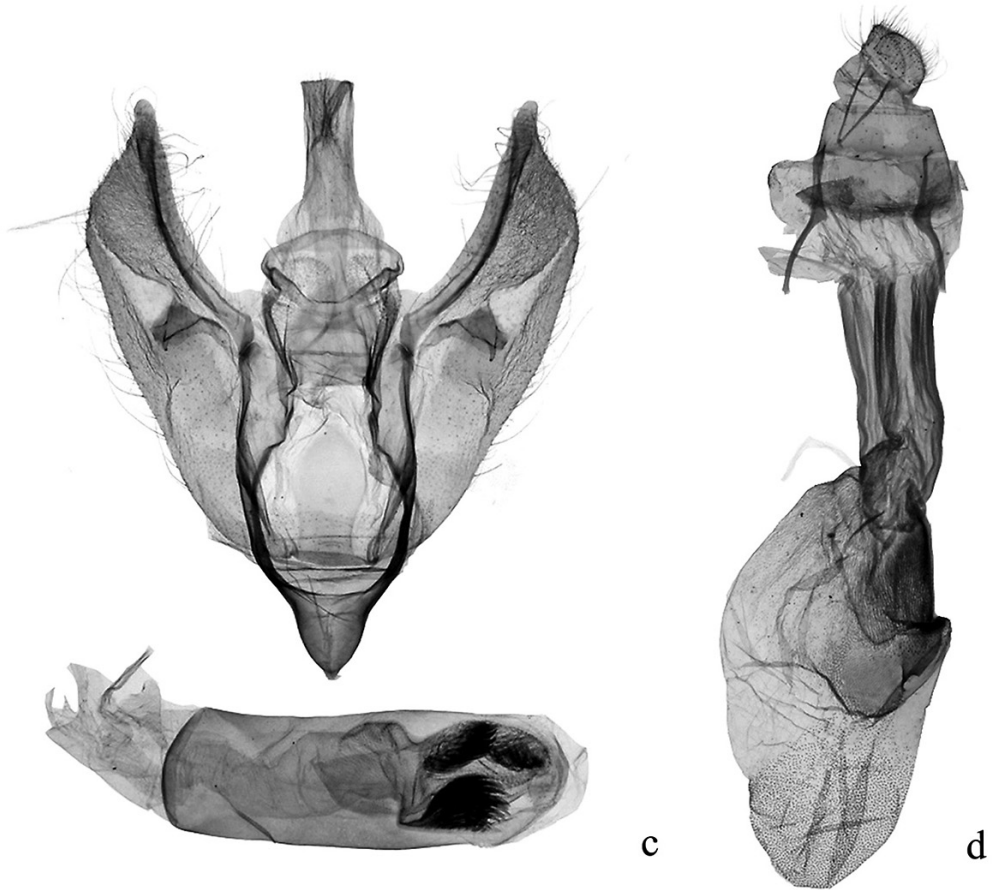

Jialiang Zhang; $1 \mathrm{o}^{\lambda}$, Huoshan County $\left(31^{\circ} 24^{\prime} \mathrm{N}\right.$, $\left.116^{\circ} 19^{\prime} \mathrm{E}\right)$, Anhui Province, 12.VIII.2004, leg.

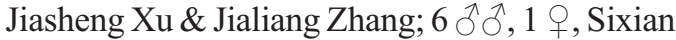
$\left(33^{\circ} 29^{\prime} \mathrm{N}, 117^{\circ} 54^{\prime} \mathrm{E}\right)$, Anhui Province, $700 \mathrm{~m}$,
15.VIII.2004, leg. Jiasheng Xu; $1 \stackrel{\jmath}{\jmath}$, Shenlongjia $\left(31^{\circ} 44^{\prime} \mathrm{N}, 110^{\circ} 40^{\prime} \mathrm{E}\right)$, Hubei Province, 1,2001,400 m, 16.VII.2003, leg. Shulian Hao; 1 ๙ै, Shenlongjia $\left(31^{\circ} 44^{\prime} \mathrm{N}, 110^{\circ} 40^{\prime} \mathrm{E}\right)$, Hubei Provin- 
ce, 1,100 m, 18.VII. 2003, leg. Shulian Hao; 1 +, Hechuan (29 $\left.58^{\prime} \mathrm{N}, 1^{\circ} 106^{\circ} 16^{\prime} \mathrm{E}\right)$, Chongqing, 3.VII.2009, leg. Xicui Du; 13 ๙ิ ฮิ, Simian Mountain $\left(28^{\circ} 39^{\prime} \mathrm{N}, 106^{\circ} 24^{\prime} \mathrm{E}\right)$, Chongqing, 1,250 m, 14.-15.VIII. 2011, leg. Guiqing He \& Lifang Song; 3 ○े $\partial^{\lambda}$, Simian Mountain $\left(28^{\circ} 39^{\prime} \mathrm{N}, 106^{\circ}\right.$ 24'E), Chongqing, 1,280 m, 1.VIII.2011, leg.

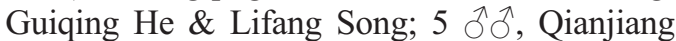
$\left(29^{\circ} 32^{\prime} \mathrm{N}, 108^{\circ} 45^{\prime} \mathrm{E}\right)$, Chongqing, 1,100 m, 25.26.VII.2012, leg. Jun Zhang \& Lijun Xun; 1 क, Qianjiang (29³2'N, $\left.108^{\circ} 45^{\prime} \mathrm{E}\right)$, Chongqing, 870 $\mathrm{m}, 23$.VII.2012, leg. Jun Zhang \& Lijun Xun; 1 `, Lu Xian (2909’N, $\left.105^{\circ} 22^{\prime} \mathrm{E}\right)$, Sichuan, Province, 24.VII.1995, leg. Yongxing Zeng; 1 , Tongjiang ( $\left.31^{\circ} 54^{\prime} \mathrm{N}, 107^{\circ} 14^{\prime} \mathrm{E}\right)$, Sichuan, Province, 400 m, 5.IX.2012, leg. Lijun Xu; 23 ふ઼ે, Kuankuoshui $\left(28^{\circ} 14^{\prime} \mathrm{N}, 107^{\circ} 10^{\prime} \mathrm{E}\right)$, Guizhou Province, 800 m, 10.-13.VIII.2010, leg. Xicui

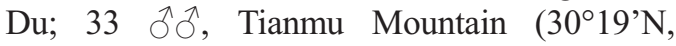
$\left.119^{\circ} 26^{\prime} E\right)$, Zhejiang Province, 400 m, 25.VII.2.VIII.2011, leg. Xicui Du \& Xiaobing Fu.

Redescription. Adult (Fig. 1c): Wingspan 18.0-24.0 mm. Body pale yellow or pale ochreous, frons white. Antenna with scape white, flagellum pale yellow or ochreous. Male antenna with ventral cilia about as long as diameter of flagellomere. Labial palp pale yellow or yellowishwhite, $1^{\text {st }}$ segment brown distally. Maxillary palp slim, pale yellow. Patagium, tegula and dorsal thorax pale yellow or pale ochreous. Forewing pale yellow or pale ochreous, with a basal black point; antemedial line represented by black or fuscous spots, obvious only in discal cell and on posterior margin; a black spot below discal cell near base; orbicular stigma and discoidal stigma black; a series of short black streaks along vein excurved round end of discal cell, then incurved at $\mathrm{CuA}_{1}$ to anal area; postmedial line represented by a series of black or fuscous spots, excurved from costa to $\mathrm{CuA}_{1}$, then bent inward to below end of discal cell, with dark mark beyond it near tornus; some indistinct fuscous streaks in terminal interspaces; cilia pale yellow. Hindwing yellowish-white, semihyaline; subterminal line indistinct, consisting of a series of small fuscous points, slightly curved; terminal pale yellow, brown near tornus; cilia yellowish-white. Legs yellowish-white, each segment of tarsus with black ringed stripe distally; fore coxa and fore femur striped with black or fuscous on outer side, fore tibia black distally; hind tarsus with inner side of first segment black. Abdomen pale yellow or pale ochreous dorsally, segments with black or fuscous spot basally except on $1^{\text {st }}$ segment, indistinct on terminal segment in some specimens; yellowish-white ventrally, with fine black or fuscous streak forming an interrupted middle line except terminal segment with a black drop basally. Some specimens without any ventral streak on terminal two segments of abdomen.

Male genitalia (Fig. 3a). Uncus short and broad, concave medially, with thin, long hairs laterally, lip-like together with vestigial gnathos band. Valva elliptic; posterior margin of valva with basal 2/3 arched, bearing a developed bundle of long hairs at middle. Costa curved toward anterior margin distally. Distal valva with a slightly sclerotized dense hairy band. Clasper near middle of valva, flat, terminal slightly concave, forming two lateral projections. Saccus broad, bluntly rounded on anterior margin. Juxta rhombic. Phallus stout; cornutus near apex, flabelliform, densely with small spines.

Female genitalia (Fig. 3b). Apophysis anterioris nearly as long as apophysis posterioris, expanded and acutely projected at about basal 1/3. Ductus bursae short and broad, about $1 / 2$ length of corpus bursae. Corpus bursae oval, contracted medially, sclerotized and with dense fine points posteriorly. Signum two, small, oval, surrounded by many smaller, sclerotized fine points.

Distribution. China (Heilongjiang, Liaoning, Tianjing, Hebei, Shaanxi, Gansu, Henan, Anhui, Hubei, Chongqing, Sichuan, Guizhou, Zhejiang, Fujian, Guangdong, Hainan), Korea, Russia.

Biology. Life history and juvenile stages unknown.

\subsection{Tabidia obvia sp. $\mathrm{n}$.}

Fig. 1d, Figs. 3c-d

Type material. Holotype. ふ઼, CHINA: Wufeng Tujiazu Autonomous County $\left(30^{\circ} 12^{\prime} \mathrm{N}, 116^{\circ}\right.$ 40’E), Hubei Province, 1,100 m, 11.VII.1999, leg. Houhun Li et al., genitalia slide No. DXC07196. Deposited in NKUM. Paratypes: CHINA: $6 \hat{\jmath} \widehat{0}$, Wufeng Tujiazu Autonomous County $\left(30^{\circ} 12^{\prime} \mathrm{N}\right.$, $\left.116^{\circ} 40^{\prime} \mathrm{E}\right)$, Hubei Province, 1,000-1,100 m, 8.12.VII.1999, leg. Houhun Li et al., [NKUM]; 1 §, 
Xingdou Mountain $\left(30^{\circ} 01^{\prime} \mathrm{N}, 1^{\circ} 9^{\circ} 06^{\prime} \mathrm{E}\right)$, Enshi, Hubei Province, 780 m, 31.VII.2012, leg. Jun Zhang \& Xiaobing Fu, [SWUCPP]; 1 $\sigma^{\lambda}$, Wen County (32 $\left.58^{\prime} \mathrm{N}, 104^{\circ} 41^{\prime} \mathrm{E}\right)$, Gansu Province, 860 m, 9.VII.2005, leg. Haili Yu, [NKUM]; 2 ठ઼ Simian Mountain $\left(28^{\circ} 39^{\prime} \mathrm{N}, \quad 106^{\circ} 24^{\prime} \mathrm{E}\right)$, Chongqing, 1,000 m, 18., 21.VII.2010, leg. Xicui Du \& Lifang Song, [SWUCPP]; 1 ô, Simian Mountain $\left(28^{\circ} 39^{\prime} \mathrm{N}, 106^{\circ} 24^{\prime} \mathrm{E}\right)$, Chongqing, 1,280 m, 11.VIII.2011, leg. Guiqing He \& Lifang Song, [SWUCPP]; 1 त, Emei Mountain (29³6'N, $\left.103^{\circ} 28^{\prime} \mathrm{E}\right)$, Sichuan Province, $550 \mathrm{~m}$, 10.VII.2011, leg. Jianbo Cao, [SWUCPP]; 1 , Mayanghe $\left(28^{\circ} 32^{\prime} \mathrm{N}, 108^{\circ} 29^{\prime} \mathrm{E}\right)$, Guizhou Province, $700 \mathrm{~m}$, 28.IX.2007, leg. Zhenhui,

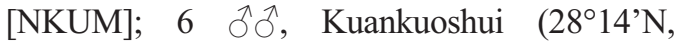
$\left.107^{\circ} 10^{\prime} \mathrm{E}\right)$, Guizhou Province, $800 \mathrm{~m}, 10 .-$ 13.VIII.2010, leg. Xicui Du, [SWUCPP]; 1 o, Kuankuoshui (28 $\left.{ }^{\circ} 14^{\prime} \mathrm{N}, 107^{\circ} 10^{\prime} \mathrm{E}\right)$, Guizhou Province, $1,500 \mathrm{~m}, 15$.VIII.2010, leg. Xicui Du, [SWUCPP]; 14 ふึَ̄, Tianmu Mountain $\left(30^{\circ} 19^{\prime} \mathrm{N}\right.$, $\left.119^{\circ} 26^{\prime} \mathrm{E}\right)$, Zhejiang Province, $400 \mathrm{~m}, 25$.VII.2.VIII.2011, leg. Xicui $\mathrm{Du} \&$ Xiaobing $\mathrm{Fu}$, [SWUCPP]; 3 ठิ๋̂, Nanling $\left(24^{\circ} 45^{\prime} \mathrm{N}\right.$, $\left.112^{\circ} 51^{\prime} \mathrm{E}\right)$, Guangdong Province, 1,070 m, 23.VIII.2010, leg. Xicui Du, [SWUCPP].

Diagnosis. This species is similar to T. strigiferalis Hampson in appearance, but can be distinguished by the postmedial line of the forewing with a distinct dentation pattern between $\mathrm{CuA}_{2}$ and $\mathrm{A}$, the subterminal line of the hindwing uninterrupted and distinct; the posterior margin of the valva slightly concave, without bundle of long hairs, but with a finger-like projection distally; three brush-like cornuti, consisting of bundles of spines. In T. strigiferalis the postmedial line of the forewing lacks the dentation pattern between $\mathrm{CuA}_{2}$ and $\mathrm{A}$, the subterminal line of the hindwing is interrupted and indistinct; the posterior margin of the valva is arched on basal 2/3 and has a developed bundle of long hairs at middle, but lacks the distal projection; the cornutus is flabelliform, with masses of small spines.

Description. Adult (Fig. 1d). Wingspan 18.0$22.0 \mathrm{~mm}$. Body pale yellow or pale ochreous, frons white. Antenna with scape white, flagellum ochreous. Male antenna with ventral cilia about two-thirds as long as the diameter of flagellomere. Labial palp pale yellow or pale ochreous except $2^{\text {nd }}$ segment brown or fuscous dorsally.
Maxillary palp slim, pale yellow. Patagium, tegula and thorax pale yellow or pale ochreous dorsally. Forewing pale yellow or pale ochreous; one indistinct brown spot below discal cell near base; antemedial line represented by fuscous spots, obvious only in discal cell and below anal vein; discoidal stigma a fuscous stripe; several short fuscous streaks round end of discal cell; postmedial line consisting of fuscous spots, excurved from costa to $\mathrm{CuA}_{1}$, then sharply inward to below end of discal cell, next sharply outward, finally curved to posterior margin, forming a distinct dentation between $\mathrm{CuA}_{2}$ and $\mathrm{A}$; some indistinct brown streaks in terminal interspaces; cilia pale yellow or pale ochreous. Hindwing yellowish-white, semihyaline; subterminal line uninterrupted and distinct between costa and $\mathrm{CuA}_{1}$; terminal pale yellow, brown between $\mathrm{CuA}_{2}$ and tornus; cilia pale yellow, yellowish-white distally. Legs yellowish-white, each segment of tarsus with fuscous ringed stripe distally; fore femur striped with pale ochreous, fore tibia grayishbrown dorsally; hind tarsus with inner side of first segment fuscous. Abdomen pale ochreous dorsally; yellowish-white ventrally, 3-6 segments with fine black or fuscous streak forming an interrupted middle line, $7^{\text {th }}$ segment with small black drop basally, terminal segment with bigger black drop basally.

Male genitalia (Fig. 3c). Uncus short and broad, slightly concave medially, with thin, long hair laterally, lip-like together with vestigial gnathos band. Valva with posterior and anterior margin nearly parallel; posterior margin of valva slightly concave, forming a finger-like projection distally. Costa slightly concave. Clasper near middle of valva, flat, with one stout spine dorsally. Saccus triangular. Juxta broad, with anterior half contracted laterally, and narrowed terminally. Phallus stout; three cornuti near apex, brush-like, consisting of bundles of spines.

Female genitalia (Fig. 3d). Apophysis anterioris about 1.5 times length of apophysis posterioris, triangularly expanded at about middle. Ductus bursae short and broad, wrinkled longitudinally, about $1 / 2$ length of corpus bursae. Corpus bursae oval, densely with fine sclerotized points and longitudinal wrinkles posteriorly, slightly sclerotized posteriorly, densely with stout spines anteriorly. 
Distribution. China (Gansu, Hubei, Chongqing, Sichuan, Guizhou, Zhejiang, Guangdong). Biology. Life history and juvenile stages unknown.

Remarks. Interrupted middle line on ventral abdomen is indistinct in some specimens.

Etymology. The specific name is derived from Latin obvius $=$ obvious, in reference to the distinct subterminal line of the hindwing.

\subsection{Key to species of Tabidia from China}

1. Body and wings white; forewing with some black blotches, basal area tinged with greyishfuscous, lower third of median area fulvous; hindwing with outer third interruptedly blackish

T. candidalis

- Body and forewings pale yellow or pale ochreous; forewing with some black or fuscous spots and streaks; hindwing yellowish-white, with an obvious or indistinct subterminal line

2. Subterminal line of hindwing interrupted and indistinct; posterior margin of valva with basal 2/3 arched, bearing a developed bundle of long hairs at middle; costa distally curved toward anterior margin

T. strigiferalis

- Subterminal line of hindwing uninterrupted and distinct; posterior margin of valva slightly concave, without bundle of long hairs; costa slightly concave

T. obvia sp.n.

Acknowledgements. We are grateful to Kevin Tuck and relevant staff (the Natural History Museum, London) for providing access to the first author examining specimens, to Matthias Nuss and Richard Mally (Senckenberg Naturhistorische Sammlungen Dresden Museum für Tierkunde, Dresden) for verifying information of Tabidia, and to Klaus Sattler (the Natural History Museum, London) for his help in translating the original description in Dutch. We also give our special thanks to Alma Solis (USDA, Smithsonian Institution) and an anonymous referee for reviewing the manuscript. The research was supported by the National Natural Science Foundation of China (31101663) and the Fundamental Research Funds for the Central Universities (XDJK2010C006).

\section{References}

Bae, Y. S., Byun, B. K. \& Paek, M. K. 2008: Pyralid moths of Korea (Lepidoptera: Pyraloidea). - Korea National Arboretum. 426 pp. [In Korean.]
Caradja, A. 1925: Ueber Chinas Pyraliden, Tortriciden, Tineiden nebst kurzen Betrachtungen, zu denen das Studium dieser Fauna Veranlassung gibt. (Eine biogeographische skizze). - Memoriile Sectiunii Stiintifice. Academia Romana (3) 3(7): 257-383.

Caradja, A. \& Meyrick, E. 1933: Materilien zu einer Microlepidopteren-Fauna kwangtungs. - Deutsche entomologische Zeitschrift Iris, Dresden 47: 156-167.

Hampson, G. F. 1896: The Fauna of British India, including Ceylon and Burma. Moths, Vol. 4. - Taylor and Francis, London. 594 pp.

Hampson, G. F. 1898: A revision of the moths of the subfamily Pyraustinae and family Pyralidae. - Proceedings of the Zoological Society of London 66: 590 761.

Hampson, G. F. 1900: New Palaearctic Pyralidae. Transactions of the Entomological Society of London 15: 369-401, pl. 3.

Kirpichnikova, V. A. 1999: Pyraloidea, — In: Ler, P. A. (ed.), Key to the insects of Russian Far East, Vol. V. Trichoptera and Lepidoptera, Pt. 2: 320-496. Vladivostok. $670 \mathrm{pp}$.

Klima, A. 1939: Pyralidae: subfamily: Pyraustinae I. — In: Bryk, F. (ed.), Lepidopterorum Catalogus 89: 1-224. W. Junk, 's-Gravenhage.

Kristensen, N. P. 2003: Skeleton and muscles: adults. In: Fischer, M. (ed.), Handbook of Zoology, Arthropoda: Insecta. Walter de Gruyter, Berlin, New York 4(36): 39-131.

Li, H. H. \& Zheng, Z. M. 1996: Methods and techniques of specimens of Microlepidoptera. - Journal of Shaanxi Normal University (Natural Science Edition) 24(3): 63-70. [In Chinese.]

Lu, J. R. \& Guan, Z. H. 1953: List of Chinese pyralid insects, addendum of $\mathrm{Hu}$ "List of Chinese Insects", part 2. Nymphulinae Fernald 1902, Scopariinae Ragonot 1890, Pyraustinae Ragonot 1891 — Acta Entomologica Sinica 3(2): 203-244. [In Chinese.]

Maes, K. V. N. 1995: A Comparative morphological study of the adult Crambidae (Lepidoptera, Pyraloidea). Bulletin et Annales de la Société Royale Entomologique de Belgique 131: 383-434.

Park, K. T. 1983: Pyralidae. — In: Shin, Y. H., Park, K. T. \& Nam, S. H. (eds.), Illustrated flora and fauna of Korea. 27 Insecta (9): 298-444. Samwha Print., Seoul.

Snellen, P. C. T. 1880: Nieuwe Pyraliden op het eiland Celebes gevonden door Mr. M. C. Piepers, en beschreven. - Tijdschrift voor Entomologie 23: 198-250.

Snellen, P. C. T. 1883: Lepidoptera van Celebes verzameld door Mr. M. C. Piepers. — Tijdschrift voor Entomologie 26: 119-144, pls. 6-8.

Song, S. M. 2003: - In: Wang, J. S., Song, S. M., Wu, Y. Y. \& Chen, T. M. (eds.), Fauna of Pyralidae of Wuyishan Nature Reserve in China [M]. China Science and Technology Press, Beijing. 328 pp. [In Chinese.]

Warren, W. 1896: New species of Pyralidae from the Khasia Hills. - Annals and Magazine of Natural History, including Zoology, Botany and Geology, London (ser. 6) 18: 163-177. 